\title{
Safety and efficacy of a propofol and ketamine based procedural sedation protocol in children with cerebral palsy undergoing botulinum toxin A injections.
}

\section{Running head: propofol and ketamine usage in children with CP}

Authors: Ryan Louer, BS ${ }^{1}$, Renee C McKinney, $\mathrm{RN}^{2}$, Samer Abu-Sultaneh, $\mathrm{MD}^{2}$, Riad Lutfi, $\mathrm{MD}^{2}$, Kamal \author{
Abulebda, $\mathrm{MD}^{2}$. \\ Affiliation: \\ ${ }^{1}$ Indiana University School of Medicine, Indianapolis, IN \\ ${ }^{2}$ Division of Pediatric Critical Care Medicine, Indiana University School of Medicine, Riley Hospital for \\ Children at Indiana University Health, Indianapolis, IN
}

Senior author: Dr. K. Abulebda, Division of Pediatric Critical Care Medicine, 705 Riley Hospital Drive, Indianapolis, IN, 46202, USA. Kabulebd@iupui.edu. Phone: 317-948-7185. Fax: 317-944-7267.

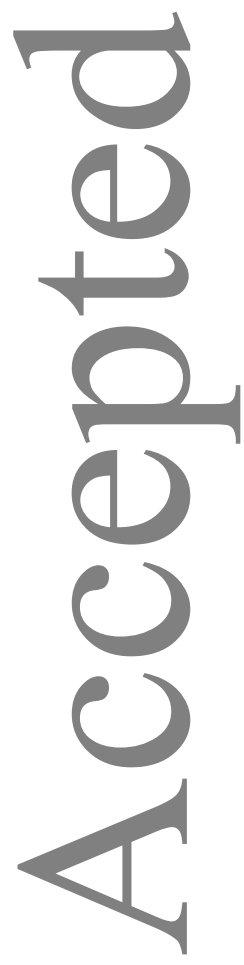

Funding: This research did not receive any specific grant from funding agencies in the public, commercial, or not-for-profit sectors.

This is the author's manuscript of the article published in final edited form as:

Louer, R., McKinney, R. C., Abu-Sultaneh, S., Lutfi, R., \& Abulebda, K. (2019). Safety and efficacy of a propofol and ketamine based procedural sedation protocol in children with cerebral palsy undergoing botulinum toxin A injections. PM\&R, 0(ja). https://doi.org/10.1002/pmrj.12146 


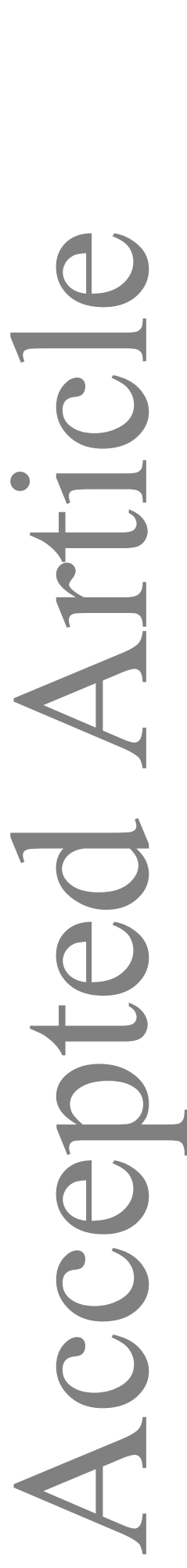
Abstract
Background: Pediatric patients with cerebral palsy (CP) often undergo intramuscular botulinum toxin (BoNT-A) injections. These injections can be painful and may require procedural sedation. An ideal sedation protocol has yet to be elucidated.
Objective: To investigate the safety and efficacy of a propofol and ketamine based sedation protocol in pediatric patients with cerebral palsy requiring BoNT-A injections.
Design: This is a retrospective chart review of children with CP undergoing propofol and ketamine based sedation for injections with botulinum toxin A.

Setting: The sedations took place in a procedural sedation suite at a tertiary children's hospital from Feb 2013 through Sept 2017.

Patients: 164 patients with diagnoses of cerebral palsy were included in this study.

Methods: An initial bolus of $0.5 \mathrm{mg} / \mathrm{kg}$ ketamine followed by a $2 \mathrm{mg} / \mathrm{kg}$ bolus of propofol was administered with supplemental boluses of propofol as needed to achieve deep sedation during the intramuscular BoNT-A injections.

Main Outcome Measurements: Propofol dosages, adverse events, serious adverse events, and sedation time parameters were reviewed.

Results: 345 sedations were successfully performed on 164 patients. The median total dose of propofol was $4.7 \mathrm{mg} / \mathrm{kg}$ (IQR 3.5, 6.3). Adverse events were encountered in $10.1 \%$ of procedures including hypoxemia responsive to supplemental oxygen $(9.6 \%)$ and transient apnea $(1.4 \%)$. The mean procedure time, recovery time and total sedation time were 10,11 and 33 minutes, respectively. With regard to patient variables, including age, weight, dose of propofol, sedation time, and Gross Motor Function Classification System classification, there was no association with increased incidence of adverse events.

Conclusion: Our sedation protocol of propofol and ketamine is safe and effective in children with cerebral palsy undergoing procedural sedation for intramuscular injections with BoNT-A. The adverse events encountered appeared to be related to airway and respiratory complications secondary to musculoskeletal deformities, emphasizing the importance of airway monitoring and management in these patients.

\section{Level III}

Key words: Cerebral palsy; pediatrics; propofol; procedural sedation; developmental disorders 


\section{Introduction}

Cerebral palsy $(\mathrm{CP})$ is a syndrome that involves an early insult to the developing central nervous system resulting in a wide variety of clinical presentations including spasticity, contracture, and dyskinesia. It is often associated with impairments in learning ability and communication ${ }^{1}$. Children with $\mathrm{CP}$, due to their motor involvement and ongoing spasticity, often require intermittent procedural interventions. Intramuscular botulinum toxin A (BoNT-A) injections are commonly indicated in these patients. The injections are often painful and frequently require some form of sedation and analgesia to reduce the associated pain, anxiety and motor disturbances ${ }^{2-4}$.

Children with CP are at an increased risk for anesthetic complications compared to healthy children as they are usually at a minimum ASA Class II, referring to mild systemic disease, by the American Society of Anesthesiologists ${ }^{5}$. These children are at increased risk of musculoskeletal deformities, such as contractures and kyphoscoliosis, which, over time, lead to restrictive lung disease ${ }^{6-8}$. Furthermore, $\mathrm{CP}$ also is associated with upper airway obstruction, swallowing dysfunction, gastroesophageal reflux, inefficient cough, and chronic airway colonization with pathogens ${ }^{6,9-13}$.

Few sedative agents, such as nitrous oxide and benzodiazepines, have been used with variable efficacy in this patient population ${ }^{14}$. Chow and Choong used a ketamine-centered protocol with success in children with CP with an adverse event rate of $6.6 \%{ }^{15}$. However, they reported that two hours of monitoring their patients after the procedure was required. The ideal sedative protocol in pediatric procedural sedation is one that is safe, effective and demonstrates rapid onset and rapid recovery ${ }^{16}$; this ideal protocol remains elusive in this patient population.

Propofol is a widely used hypnotic agent in pediatric patients for procedural sedation. It is often selected due to its rapid onset of action, efficacy in achieving sedation, and rapid emergence from sedation ${ }^{17}$. Small doses of adjunctive ketamine $(0.5 \mathrm{mg} / \mathrm{kg})$ have been shown to produce anxiolysis, reduce the total dose of propofol required to achieve adequate sedation, and preserve cardiopulmonary function ${ }^{18-23}$. The use of propofol in children with $\mathrm{CP}$ for MRI and other procedures has been sparsely documented, and published 
reports with regards to safety and efficacy of a procedural sedation protocol to facilitate BoNT-A injections $\operatorname{are~lacking~}^{24-25}$.

The aim of this study is to investigate the safety and efficacy of a propofol and ketamine based sedation protocol in pediatric patients with cerebral palsy receiving BoNT-A injections.

\begin{abstract}
Methods
This retrospective chart review was approved by the Indiana University institutional review board. Children with $\mathrm{CP}$ who underwent procedural sedation with propofol and ketamine for BoNT-A injections to treat spasticity between February 2013 and September 2017 were included in the analysis. All patients with cerebral palsy had a formal primary diagnosis made by their neurologist and this information was provided on the request form by the physician making the referral for BoNT-A. Exclusion criteria included patients who were admitted to the hospital at the time of their scheduled study for reasons other than BoNT-A injections and patients who were on digoxin or B-blockers. Patient demographics, incidence of adverse events and serious adverse events, sedative drug dosages, and procedure, sedation, recovery and discharge times were collected. Patients were classified according to the Gross Motor Function Classification System (GMFCS), which is a standardized five level classification system (I to V) to classify the gross motor function of children with cerebral palsy ${ }^{26}$.
\end{abstract}

Our institution has an intensivist-based procedural sedation program that adheres to policies and guidelines based on recommendations by the Joint Commission and the American Academy of Pediatrics ${ }^{27-28}$. Oral and enteral intake were withheld for at least 6 hours prior to the onset of the procedure. Patients are prescreened via telephone interview by a sedation nurse with a parent/guardian present during the interview and by reviewing the chart filled out by the primary physician.

Our sedation team used a standard approach for sedation with propofol and ketamine. An initial bolus of $0.5 \mathrm{mg} / \mathrm{kg}$ of ketamine was administered intravenously followed by a $2 \mathrm{mg} / \mathrm{kg}$ bolus of intravenous propofol. If unwanted movement occurred, additional boluses of $0.5-1 \mathrm{mg} / \mathrm{kg}$ of propofol were given to 
achieve the desirable level of sedation. The sedation level of the children was measured by the sedation team using the Ramsay sedation scale every 5 minutes. The Ramsay scale assigns a score of $1-6$ based on the clinical assessment of the level of sedation as follows: (1) anxious, agitated, restless; (2) awake, but cooperative, tranquil, oriented and (3) responds to verbal commands only. Scores 4 to 6 were used for sleeping patients and are graded according to the response to loud noises or glabellar taps as follows: (4) brisk response; (5) sluggish response and (6) no response ${ }^{29}$. Score 4 and above were accepted as deep sedation. A standardized dose of 12 international units $/ \mathrm{kg}$, up to a maximum dose of $400 \mathrm{IU}$, of BotNT-A was ordered by the physician in charge of administering the BoNT-A using electronic medical record order sets. The exact administered dosage is carefully determined by the physician and can be affected by level of spasticity and number of the injected muscles.

Standardized monitoring, in accordance with the AAP 2016 guidelines, is used at our institution for procedural sedation, which includes, at a minimum, baseline vitals (including temperature), continuous $\mathrm{SaO}_{2}$, heart rate and ventilation monitoring, and automatic blood pressure checks every 5 minutes ${ }^{28}$. Adverse events were defined as development of transient hypoxemia (oxygen saturation of less than $90 \%$ for 30 seconds), hypotension (drop in systolic blood pressure [SBP] below expected age appropriate normal range or dropping by $20 \%$ from starting SBP), transient apnea, nausea and vomiting. Serious adverse events such as endotracheal intubation, respiratory or cardiac arrest, failure to complete the procedure, and transfer to higher level care were also recorded. Procedure time (PT) was defined as the time between the first dose of propofol until the procedure was completed. Recovery time (RT) was defined as the interval between the end of the procedure until the patient's level of consciousness returned to Ramsay level 2. Total time was defined as the time recorded by the nurse assisting with the sedation from the first dose until the patient was ready for discharge.

\section{Statistical analysis:}

Overall cohort demographics, incidence of adverse events, and sedation time parameters are presented as median (IQR) for continuous variables, and frequencies (percent) for categorical variables. Age, weight, GMFCS score, ketamine dose, propofol dose, and sedation time parameters were compared for each of the 
aforementioned adverse events versus those without the events using Wilcoxon rank-sum tests or Fisher's exact test as appropriate.

\section{Results}

A total of 345 sedation encounters in 164 patients were performed successfully using propofol and ketamine according to our standardized protocol. During the study period, all sedation encounters were successfully completed. Patient demographics are shown in Table 1. Most patients (97.6\%) were of ASA II category. Out of 164 patients, 138 patients (84.1\%) were GMFCS III and higher. The median total dose of propofol for each procedure was $4.7 \mathrm{mg} / \mathrm{kg}$ (IRQ 3.5,6.3). The number of sedations per patient during the study period are shown in figure 1. Thirty-five patient encounters (10.1\%) out of 345 experienced adverse events, of which 33 had hypoxemia (9.6\%), 5 (1.4\%) had transient apnea, and $3(0.9 \%)$ had both. All episodes of hypoxemia and apnea were transient and resolved with only supplemental oxygen via nasal cannula. There were no serious adverse events (Table 2). Average procedure time, recovery time and total time were 11, 10 and 33 minutes, respectively (Table 3). Patient age, patient weight, dose of propofol, procedure time, recovery time, and total nurse time did not vary significantly between the patient groups with and without adverse events (Tables 4 and 5). Additionally, incidence of adverse events and hypoxemia were not significantly different between the GMFCS classes.

\section{Discussion}

Pediatric patients undergoing procedural sedation require an effective sedation protocol with agents that have a rapid onset, a rapid recovery, and a favorable side effect profile. Children with cerebral palsy requiring BoNT-A injections present unique challenges due to their developmental abnormalities and the pain and distress that can be associated with these procedures ${ }^{30}$. Our study showed that a propofol and ketamine based sedation protocol is both safe and effective when conducted in an appropriate setting with providers highly trained in pediatric airway and cardiorespiratory monitoring. We demonstrated that $100 \%$ of our patients were successfully sedated using our protocol with a mean procedure time of 11 minutes, a mean recovery time of 10 minutes, and a mean total time of 33 minutes. In terms of safety, the overall incidence of adverse events was $10.1 \%$ in our patient population, of which hypoxemia responsive to 
supplemental oxygen via nasal cannula was the vast majority (9.6\%), while transient apnea occurred in $1.4 \%$. We also found that GMFCS classification had no significant impact on adverse events in these patients.

Several studies have demonstrated the benefits of BoNT-A injections in patients with CP as well as its functional outcomes ${ }^{31-32}$. However, little work has been done in regard to regimens to alleviate the pain and distress that result from these procedures. Zeir et al compared inhaled nitrous oxide to enteral midazolam and demonstrated suboptimal efficacy of both regimens in providing analgesia and desired level of sedation to pediatric patients undergoing BoNT-A injections ${ }^{33}$.

At our institution, we use an adjunctive dose of ketamine prior to propofol administration. This approach in anxiolysis prior to sedation has been widely studied and the effects of ketamine are well known throughout pediatric patient sub-populations ${ }^{18-20}$. Ketamine has also been shown to reduce the dose of propofol required for sedation, while providing cardiovascular stability and preserving a patient maintained airway ${ }^{21-23}$. Propofol has been studied and shown to be safe and effective in pediatric patients undergoing a variety of procedures, like transesophageal echocardiography and magnetic resonance imaging, in both inpatient and outpatient settings ${ }^{34-36}$. More importantly, combining propofol and ketamine has been evaluated in large case studies in patients with a variety of primary diagnoses, which include hematologic, oncologic, infectious, neurologic and many other diagnoses ${ }^{21,37}$. This combination of agents has been studied in the emergency department, radiological imaging units and ICU settings and has been found to be safe and effective when administered by skilled personnel, while also resulting in a more rapid recovery, shorter stay and smoother anesthetic emergence ${ }^{21,37-40}$.

Since several of these procedures are often performed in a day, and because patients are routinely discharged directly home after the intervention, it is important to have a regimen that allows for rapid onset of induction and rapid recovery from sedation. We attribute the speed of recovery and discharge in our study to the pharmacologic properties of propofol, specifically its rapid redistribution and clearance from circulation $^{41-43}$. These properties make propofol an ideal agent to be used in the outpatient setting if used by 
qualified physicians trained in sedation and advanced airway management ${ }^{44-45}$. The Chow and Choong study proposing the use of ketamine and midazolam, and later on ketamine alone, to provide sedation in pediatric patients with $\mathrm{CP}$ undergoing BoNT-A injections found that those patients required monitoring for two hours post-procedure ${ }^{15}$. This is much longer than the mean total procedure time and mean recovery time in our study. We believe that this is likely due to the relatively longer half-life of ketamine compared to propofol, particularly when higher doses of ketamine are used. The shorter recovery time using our propofol and ketamine based protocol ultimately improved patient throughput and the overall work flow of the sedation team.

In terms of safety and adverse events, the overall incidence of adverse events and incidence of hypoxemia were $10.1 \%$ and $9.6 \%$ respectively. This is higher that the incidence of adverse events of $5 \%$ reported by the Pediatric Sedation Research Consortium data using propofol for sedation in the general pediatric population ${ }^{17}$. In that study, and others, propofol has been shown to be associated with airway obstruction, desaturation, and coughing and increased secretions among other adverse events ${ }^{17,46}$. However, children with $\mathrm{CP}$ are at increased risk of desaturation due to higher rates of restrictive lung diseases, muscle contractures, increased salivation and higher incidence of gastroesophageal reflux ${ }^{6-13}$. In our patients, there was no difference in adverse events between any of the GMFCS classification groups. This could be due to the fact that all sedations were conducted in a consistent location with a dedicated sedation team. This is an important finding as it highlights the role of having a skilled sedation staff who were aware of potential sedation-related adverse events and having the appropriate equipment readily available in providing equally safe and effective procedural sedation to a spectrum of children with CP with different GMFCS classifications. In comparing our study to the recent Chow and Choong study, the adverse event rate reported for their study was $6.6 \%$ in a patient population where only $40.2 \%$ of patients were classified as a GMFCS of 3 or greater; adverse events reported included rash, nausea and vomiting, tremors, headache, and nightmares. We hypothesize that the difference in types of adverse events experienced between our study and theirs is mainly due to the pharmacologic agent used while the incidence of adverse events is possibly related to a patient population with a higher disease severity based on GMFCS classification. No serious adverse events were encountered in our study population.

This article is protected by copyright. All rights reserved. 
Our study has a few limitations. It is retrospective in nature and was conducted at a single center with a relatively small number of patients. Additionally, our sedations were performed by a pediatric intensivistbased sedation team, which may limit the generalizability of these findings to other health care providers and settings when performing procedural sedation. However, due to the standardized protocol used, and the similar safety profile among all children with $\mathrm{CP}$ with different complexity levels, it can be argued that our protocol can be utilized by other teams with different backgrounds as long as team providers are adequately trained in pediatric airway and cardiorespiratory monitoring and management. We are able to report that, based on our study, our protocol using propofol and ketamine is quite safe and highly efficacious in sedating pediatric patients with cerebral palsy regardless of the severity of their underlying $\mathrm{CP}$.

\section{Conclusion}

In summary, we conclude that a propofol and ketamine based sedation protocol is both a safe and an effective method to provide deep sedation for pediatric patients with cerebral palsy undergoing intramuscular BoNT-A injections. Furthermore, we believe that this protocol could be utilized to provide sedation for other non-invasive and minimally invasive procedures in children with cerebral palsy. Given the higher incidence of adverse events in this particular patient population, these patients require vigilant monitoring by providers adequately trained in pediatric airway and cardiorespiratory monitoring.

\section{References:}

1. $\quad$ Colver, A.; Fairhurst, C.; Pharoah, P. O., Cerebral palsy. Lancet 2014, 383 (9924), 1240-9.

2. $\quad$ Forrester, M.; Srinivasan, J.; Mihrshahi, S.; Waugh, M.; O'Flaherty, S.; Rice, J.; Graham, K.; Scheinberg, A., Conscious sedation or general anaesthetic for intramuscular botulinum toxin injections in children - a two centre cross-sectional prospective audit. Eur J Paediatr Neurol 2012, 16 (2), 215-7.

3. Carraro, E.; Trevisi, E.; Martinuzzi, A., Safety profile of incobotulinum toxin A [Xeomin((R))] in gastrocnemious muscles injections in children with cerebral palsy: Randomized double-blind clinical trial. Eur J Paediatr Neurol 2016, 20 (4), 532-7.

4. $\quad$ Edwards, P.; Sakzewski, L.; Copeland, L.; Gascoigne-Pees, L.; McLennan, K.; Thorley, M.; Kentish, M.; Ware, R.; Boyd, R. N., Safety of Botulinum Toxin Type A for Children With Nonambulatory Cerebral Palsy. Pediatrics 2015, 136 (5), 895-904.

5. ASA Physical Status Classification System. https://www.asahq.org/ /media/sites/asahq/files/public/resources/standards-guidelines/asa-physical-statusclassification-system.pdf (accessed November 11).

6. Proesmans, M., Respiratory illness in children with disability: a serious problem? Breathe (Sheff) 2016, 12 (4), e97-e103. 
7. Chan, G.; Miller, F., Assessment and treatment of children with cerebral palsy. Orthop Clin North Am 2014, 45 (3), 313-25.

8. Noble-Jamieson, C. M.; Heckmatt, J. Z.; Dubowitz, V.; Silverman, M., Effects of posture and spinal bracing on respiratory function in neuromuscular disease. Arch Dis Child 1986, 61 (2), 178-81.

9. Kotagal, S.; Gibbons, V. P.; Stith, J. A., Sleep abnormalities in patients with severe cerebral palsy. Dev Med Child Neurol 1994, 36 (4), 304-11.

10. Arvedson, J.; Rogers, B.; Buck, G.; Smart, P.; Msall, M., Silent aspiration prominent in children with dysphagia. Int J Pediatr Otorhinolaryngol 1994, 28 (2-3), 173-81.

11. Gustafsson, P. M.; Tibbling, L., Gastro-oesophageal reflux and oesophageal dysfunction in children and adolescents with brain damage. Acta Paediatr 1994, 83 (10), 1081-5.

12. Seddon, P. C.; Khan, Y., Respiratory problems in children with neurological impairment. Arch Dis Child 2003, 88 (1), 75-8.

13. Thorburn, K.; Jardine, M.; Taylor, N.; Reilly, N.; Sarginson, R. E.; van Saene, H. K., Antibioticresistant bacteria and infection in children with cerebral palsy requiring mechanical ventilation. Pediatr Crit Care Med 2009, 10 (2), 222-6.

14. Gubbay, A.; Langdon, K., 'Effectiveness of sedation using nitrous oxide compared with enteral midazolam for botulinum toxin A injections in children'. Dev Med Child Neurol 2009, 51 (6), 491-2; author reply 492.

15. Chow, C.; Choong, C. T., Ketamine-based procedural sedation and analgesia for botulinum toxin A injections in children with cerebral palsy. Eur J Paediatr Neurol 2016, 20 (2), 319-322.

16. Krauss, B.; Green, S. M., Procedural sedation and analgesia in children. Lancet 2006, 367 (9512), 766-80.

17. Kamat, P. P.; McCracken, C. E.; Gillespie, S. E.; Fortenberry, J. D.; Stockwell, J. A.; Cravero, J. P.; Hebbar, K. B., Pediatric critical care physician-administered procedural sedation using propofol: a report from the Pediatric Sedation Research Consortium Database. Pediatr Crit Care Med 2015, 16 (1), 1120.

18. Sen, S.; Thakurta, R. G.; Gupta, S. D.; Bhattacharya, S.; Mukherji, S., Preoperative anxiolysis in pediatric population: A comparative study between oral midazolam and oral ketamine. Anesth Essays Res 2013, 7 (2), 200-5.

19. McCann, M. E.; Kain, Z. N., The management of preoperative anxiety in children: an update. Anesth Analg 2001, 93 (1), 98-105.

20. Gorlin, A. W.; Rosenfeld, D. M.; Ramakrishna, H., Intravenous sub-anesthetic ketamine for perioperative analgesia. J Anaesthesiol Clin Pharmacol 2016, 32 (2), 160-7.

21. Alletag, M. J.; Auerbach, M. A.; Baum, C. R., Ketamine, propofol, and ketofol use for pediatric sedation. Pediatr Emerg Care 2012, 28 (12), 1391-5; quiz 1396-8.

22. Patino, M.; Samuels, P.; Mahmoud, M., Pediatric sedation outside the operating room. Int Anesthesiol Clin 2013, 51 (2), 127-46.

23. Mortero, R. F.; Clark, L. D.; Tolan, M. M.; Metz, R. J.; Tsueda, K.; Sheppard, R. A., The effects of small-dose ketamine on propofol sedation: respiration, postoperative mood, perception, cognition, and pain. Anesth Analg 2001, 92 (6), 1465-9.

24. Kim, E. J.; Jo, Y. Y.; Kil, H. K., Optimal sedative dose of propofol to start MRI in children with cerebral palsy. Korean J Anesthesiol 2011, 61 (3), 216-9.

25. Saricaoglu, F.; Celebi, N.; Celik, M.; Aypar, U., The evaluation of propofol dosage for anesthesia induction in children with cerebral palsy with bispectral index (BIS) monitoring. Paediatr Anaesth 2005, 15 (12), 1048-52.

26. Palisano, R.; Rosenbaum, P.; Walter, S.; Russell, D.; Wood, E.; Galuppi, B., Development and reliability of a system to classify gross motor function in children with cerebral palsy. Dev Med Child Neurol 1997, 39 (4), 214-23.

27. American Society of Anesthesiologists Task Force on, S.; Analgesia by, N.-A., Practice guidelines for sedation and analgesia by non-anesthesiologists. Anesthesiology 2002, 96 (4), 1004-17.

28. Cote, C. J.; Wilson, S.; American Academy Of, P.; American Academy Of Pediatric, D., Guidelines for Monitoring and Management of Pediatric Patients Before, During, and After Sedation for Diagnostic and Therapeutic Procedures: Update 2016. Pediatrics 2016, 138 (1).

29. Ramsay, M. A.; Savege, T. M.; Simpson, B. R.; Goodwin, R., Controlled sedation with alphaxalone-alphadolone. Br Med J 1974, 2 (5920), 656-9. 
30. Linder-Lucht, M.; Kirschner, J.; Herrmann, J.; Geth, K.; Korinthenberg, R.; Berweck, S.; Heinen, F.; Mall, V., 'Why do children with cerebral palsy discontinue therapy with botulinum toxin A?'. Dev Med Child Neurol 2006, 48 (4), 319-20.

31. Koman, L. A.; Paterson Smith, B.; Balkrishnan, R., Spasticity associated with cerebral palsy in children: guidelines for the use of botulinum A toxin. Paediatr Drugs 2003, 5 (1), 11-23.

32. Bakheit, A. M., Botulinum toxin in the management of childhood muscle spasticity: comparison of clinical practice of 17 treatment centres. Eur J Neurol 2003, 10 (4), 415-9.

33. Zier, J. L.; Rivard, P. F.; Krach, L. E.; Wendorf, H. R., Effectiveness of sedation using nitrous oxide compared with enteral midazolam for botulinum toxin A injections in children. Dev Med Child Neurol 2008, 50 (11), 854-8.

34. Chiaretti, A.; Benini, F.; Pierri, F.; Vecchiato, K.; Ronfani, L.; Agosto, C.; Ventura, A.; Genovese, O.; Barbi, E., Safety and efficacy of propofol administered by paediatricians during procedural sedation in children. Acta Paediatr 2014, 103 (2), 182-7.

35. Mart, C. R.; Parrish, M.; Rosen, K. L.; Dettorre, M. D.; Ceneviva, G. D.; Lucking, S. E.; Thomas, N. J., Safety and efficacy of sedation with propofol for transoesophageal echocardiography in children in an outpatient setting. Cardiol Young 2006, 16 (2), 152-6.

36. Meredith, J. R.; O'Keefe, K. P.; Galwankar, S., Pediatric procedural sedation and analgesia. $J$ Emerg Trauma Shock 2008, 1 (2), 88-96.

37. Grunwell, J. R.; Travers, C.; Stormorken, A. G.; Scherrer, P. D.; Chumpitazi, C. E.; Stockwell, J. A.; Roback, M. G.; Cravero, J.; Kamat, P. P., Pediatric Procedural Sedation Using the Combination of Ketamine and Propofol Outside of the Emergency Department: A Report From the Pediatric Sedation Research Consortium. Pediatr Crit Care Med 2017, 18 (8), e356-e363.

38. Phillips, W.; Anderson, A.; Rosengreen, M.; Johnson, J.; Halpin, J., Propofol versus propofol/ketamine for brief painful procedures in the emergency department: clinical and bispectral index scale comparison. J Pain Palliat Care Pharmacother 2010, 24 (4), 349-55.

39. Chiaretti, A.; Ruggiero, A.; Barbi, E.; Pierri, F.; Maurizi, P.; Fantacci, C.; Bersani, G.; Riccardi, R., Comparison of propofol versus propofol-ketamine combination in pediatric oncologic procedures performed by non-anesthesiologists. Pediatr Blood Cancer 2011, 57 (7), 1163-7.

40. Pershad, J.; Godambe, S. A., Propofol for procedural sedation in the pediatric emergency department. J Emerg Med 2004, 27 (1), 11-4.

41. Kotani, Y.; Shimazawa, M.; Yoshimura, S.; Iwama, T.; Hara, H., The experimental and clinical pharmacology of propofol, an anesthetic agent with neuroprotective properties. CNS Neurosci Ther 2008, 14 (2), 95-106.

42. Kanto, J.; Gepts, E., Pharmacokinetic implications for the clinical use of propofol. Clin Pharmacokinet 1989, 17 (5), 308-26.

43. Vardi, A.; Salem, Y.; Padeh, S.; Paret, G.; Barzilay, Z., Is propofol safe for procedural sedation in children? A prospective evaluation of propofol versus ketamine in pediatric critical care. Crit Care Med 2002, 30 (6), 1231-6.

44. $\quad$ Larsen, R.; Galloway, D.; Wadera, S.; Kjar, D.; Hardy, D.; Mirkes, C.; Wick, L.; Pohl, J. F., Safety of propofol sedation for pediatric outpatient procedures. Clin Pediatr (Phila) 2009, 48 (8), 819-23. 45. Srinivasan, M.; Turmelle, M.; Depalma, L. M.; Mao, J.; Carlson, D. W., Procedural sedation for diagnostic imaging in children by pediatric hospitalists using propofol: analysis of the nature, frequency, and predictors of adverse events and interventions. J Pediatr 2012, 160 (5), 801-806 e1.

46. Chidambaran, V.; Costandi, A.; D'Mello, A., Propofol: a review of its role in pediatric anesthesia and sedation. CNS Drugs 2015, 29 (7), 543-63. 


\section{Tables}

Table 1: Demographic characteristics of children with cerebral palsy receiving sedation with propofol and ketamine for botulinum toxin A injections.

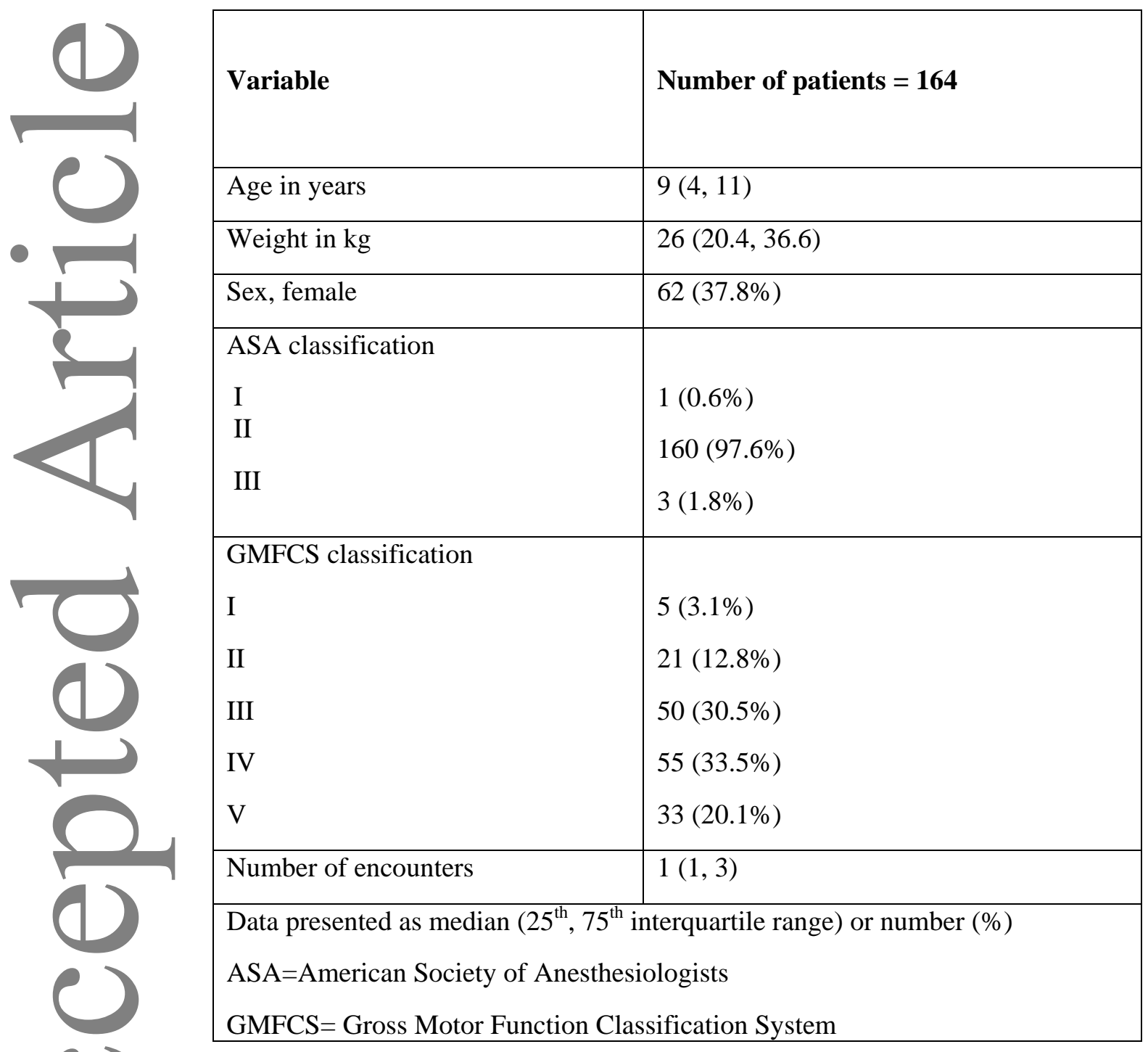

This article is protected by copyright. All rights reserved. 
Table 2: Incidence of adverse events for all procedures

\begin{tabular}{|l|l|}
\hline Adverse events & $\begin{array}{l}\text { Frequency } \\
\mathbf{N}=\mathbf{3 4 5}\end{array}$ \\
\hline Hypotension & $0(0 \%)$ \\
\hline Hypoxemia & $33(9.6 \%)$ \\
\hline Apnea & $5(1.4 \%)$ \\
\hline Nausea and Vomiting & $0(0 \%)$ \\
\hline All Adverse events & $35(10.1 \%)$ \\
\hline Serious Adverse Events & $0(0 \%)$ \\
\hline Data presented as number $(\%)$ & \\
\hline
\end{tabular}

This article is protected by copyright. All rights reserved. 
Table 3: Sedation time parameters .

\begin{tabular}{|c|c|}
\hline Variable & Time (in minutes) \\
\hline Procedure Time & $11(9,14)$ \\
\hline Recovery Time & $10(6,17)$ \\
\hline Total Sedation Time & $33(27,40)$ \\
\hline \multicolumn{2}{|c|}{$\begin{array}{l}\text { Data presented as median }\left(25^{\text {th }}, 75^{\text {th }} \text { interquartile range }\right) \text {. Data } \\
\text { presented as median }\left(25^{\text {th }}, 75^{\text {th }} \text { interquartile range }\right) .\end{array}$} \\
\hline \multicolumn{2}{|c|}{$\begin{array}{l}\text { Procedure time was defined as interval from first dose of } \\
\text { propofol to completion of injections }\end{array}$} \\
\hline \multicolumn{2}{|c|}{$\begin{array}{l}\text { Recovery time was defined as the interval from procedure end } \\
\text { time to consciousness at Ramsay level }\end{array}$} \\
\hline \multicolumn{2}{|c|}{$\begin{array}{l}\text { Total time was the sum of both procedure time and recovery } \\
\text { time. }\end{array}$} \\
\hline
\end{tabular}

This article is protected by copyright. All rights reserved. 
Table 4: Demographics and sedation time parameters for patients with and without hypoxemia

\begin{tabular}{|c|c|c|c|}
\hline Variable & $\begin{array}{c}\text { Hypoxemia } \\
(\mathrm{N}=33)\end{array}$ & $\begin{array}{c}\text { No Hypoxemia } \\
(\mathbf{N}=312)\end{array}$ & p-value \\
\hline Age in years & $5.1(3.6,6.3)$ & $5.8(3.9,9.4)$ & 0.12 \\
\hline Weight in $\mathrm{kg}$ & $19.2(15.8,22.8)$ & $20.5(16.2,27.9)$ & 0.33 \\
\hline Dose of Propofol (mg/kg) & $5.0(3.7,7.0)$ & $4.7(3.5,6.1)$ & 0.17 \\
\hline $\begin{array}{l}\text { GMFCS classification } \\
\text { I } \\
\text { II } \\
\text { III } \\
\text { IV } \\
\text { V }\end{array}$ & $\begin{array}{l}3(18.8 \%) \\
5(8.3 \%) \\
8(8.6 \%) \\
8(6.9 \%) \\
9(15.0 \%)\end{array}$ & $\begin{array}{l}13(81.3 \%) \\
55(91.7 \%) \\
85(91.4 \%) \\
108(93.1 \%) \\
51(85.0 \%)\end{array}$ & 0.33 \\
\hline Procedure Time & $13(10,16)$ & $11(9,14)$ & 0.06 \\
\hline Recovery Time & $13(7,20)$ & $10(6,15)$ & 0.09 \\
\hline Sedation Total Time & $36(32,41)$ & $33(26,40)$ & 0.07 \\
\hline
\end{tabular}

This article is protected by copyright. All rights reserved. 
Table 5: Demographics and sedation time parameters for patients with and without any complication.

\begin{tabular}{|c|c|c|c|}
\hline Variable & $\begin{array}{c}\text { Complication During } \\
\text { Sedation } \\
(\mathbf{N}=35)\end{array}$ & $\begin{array}{l}\text { No Complication } \\
\text { During Sedation } \\
\qquad(\mathrm{N}=\mathbf{3 1 0})\end{array}$ & p-value \\
\hline Age in years & $5.0(3.6,6.3)$ & $5.9(3.9,9.4)$ & 0.09 \\
\hline Weight in $\mathrm{kg}$ & $18.7(15.6,22.8)$ & $20.6(16.3,28.0)$ & 0.2 \\
\hline Dose of Propofol (mg/kg) & $5.1(3.7,7.0)$ & $4.6(3.5,6.1)$ & 0.09 \\
\hline $\begin{array}{l}\text { GMFCS classification } \\
\text { I } \\
\text { II } \\
\text { III } \\
\text { IV } \\
\text { V }\end{array}$ & $\begin{array}{l}3(18.8 \%) \\
6(10.0 \%) \\
9(9.7 \%) \\
8(6.9 \%) \\
9(15.0 \%)\end{array}$ & $\begin{array}{c}13(81.3 \%) \\
54(90.0 \%) \\
84(90.3 \%) \\
108(93.1 \%) \\
51(85.0 \%)\end{array}$ & 0.37 \\
\hline Procedure Time & $13(10,16)$ & $11(9,14)$ & 0.07 \\
\hline Recovery Time & $13(7,20)$ & $10(6,15)$ & 0.05 \\
\hline Sedation Total Time & $36(32,42)$ & $33(26,40)$ & 0.06 \\
\hline
\end{tabular}

Figure 1: Number of sedations received during the study period for patients with $\mathrm{CP}$ undergoing injections with BoNT-A.

This article is protected by copyright. All rights reserved. 


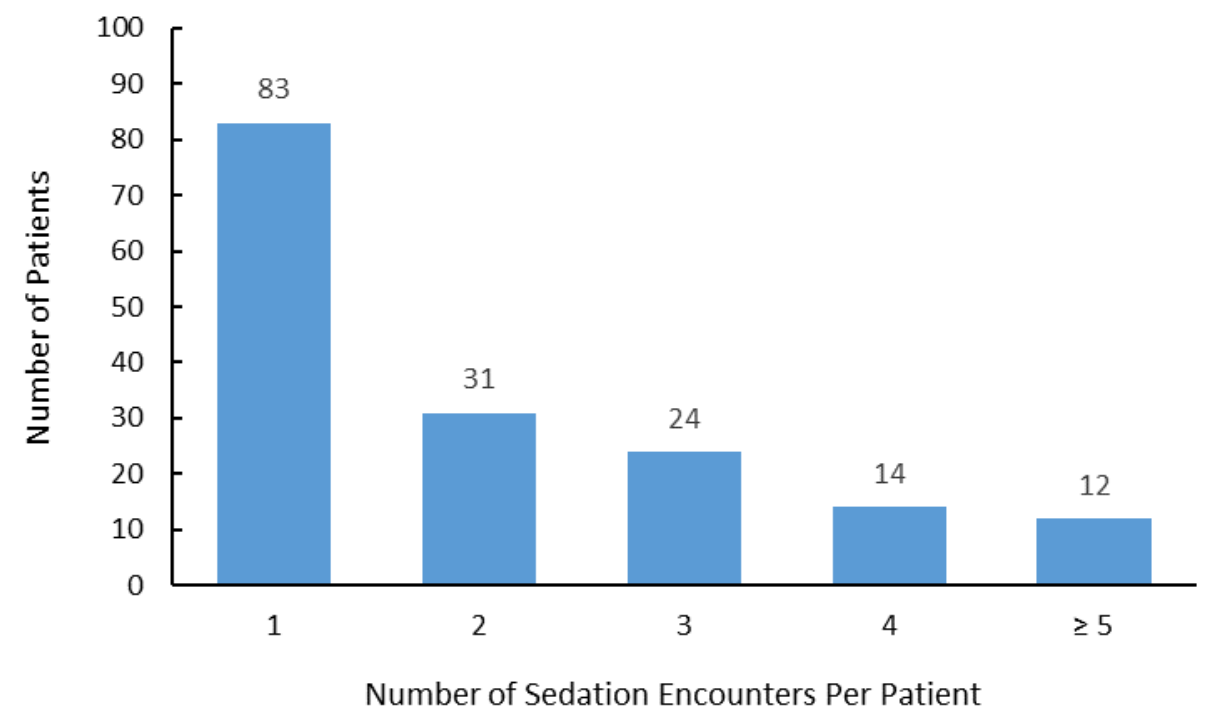

This article is protected by copyright. All rights reserved. 